\title{
The seven-layer stratification theory of gastric wall under ultrasonography and its clinical application
}

\section{Kexiao Mu ( $\nabla$ usxiran@163.com )}

Ultrasound Department, The Second Affiliated Hospital of Shandong University of Traditional Chinese Medicine, Qingdao, 250002, Shandong

\section{Qian Sun}

Physical Diagnosis Department, The West Campus of Qingdao Municipal Hospital, Qingdao, 266002, Shandong

\section{Research Article}

Keywords: Gastric wall, ultrasound, seven-layer stratification, clinical application

Posted Date: August 23rd, 2021

DOl: https://doi.org/10.21203/rs.3.rs-828840/v1

License: (1) This work is licensed under a Creative Commons Attribution 4.0 International License. Read Full License 


\section{Abstract}

Objective

Here, we develop a seven-layer gastric wall stratification theory based on the physical basis of ultrasound and histology, and further discuss its potential clinical application.

Methods

1. Experimental methods: Ex vivo human gastric specimens were immersed in normal saline and examined with a high-frequency probe to study the relationship between the sonograms and the corresponding anatomy of the gastric wall. 2. The study enrolled 136 patients admitted to our hospital with gastric diseases who underwent gastric contrast ultrasonography supplemented with the pathological examination. The seven-layer stratification theory was adopted during the analysis to profile sonogram characteristics with lesions originating from various layers.

Results

All the sonograms of the in vitro human gastric specimens could be divided into seven intervals of strong and weak echoes. The pathological examinations were performed on 136 patient-derived samples as the golden criteria of diagnosis: 29 cases of gastric polyps, 10 cases of lymphomas, 5 cases of neuroendocrine tumors, 11 cases of ectopic pancreas, 22 cases of gastric stromal tumor, 19 cases of leiomyomas, 29 cases of chronic inflammation, 9 cases of diffuse invasive cancer, and 2 cases of neurilemmoma. The ultrasound and pathological examination results were consistent in 110 cases, showing a coincidence rate of $80.9 \%$.

Conclusion

By adopting the seven-layer stratification theory of the gastric wall, the ultrasound can accurately locate the position of mucosal muscularis, which is of great significance for accurate measurement of the thickness of each anatomical layer and the correct judgment of the origin and the classification of the space-occupying lesions. Keywords Gastric wall; ultrasound; seven-layer stratification; clinical application

\section{Full Text}

This preprint is available for download as a PDF.

\section{Figures}



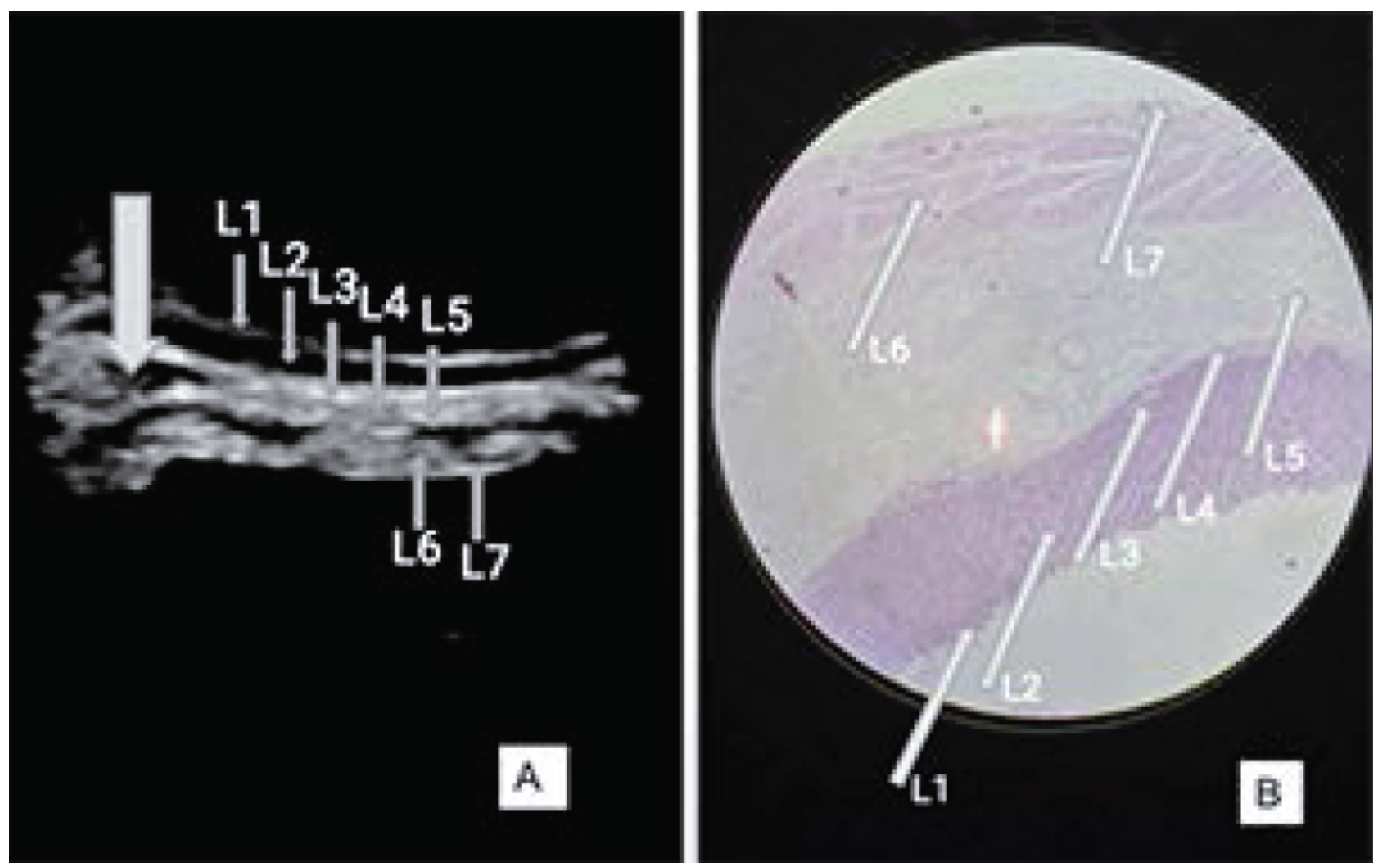

\section{Figure 1}

The histological structure of the gastric wall in sonograms(A) and microscope(B). (A) The thick white arrow shows the separation of the mucosal layer from the submucosa. (B) Isolated stomach as seen under ordinary light microscope, magnification $(4 \times 10)$. Because the submucosal layer is loosely organized and easy to enter air, the L5 is thicker than the normal gastric wall. 


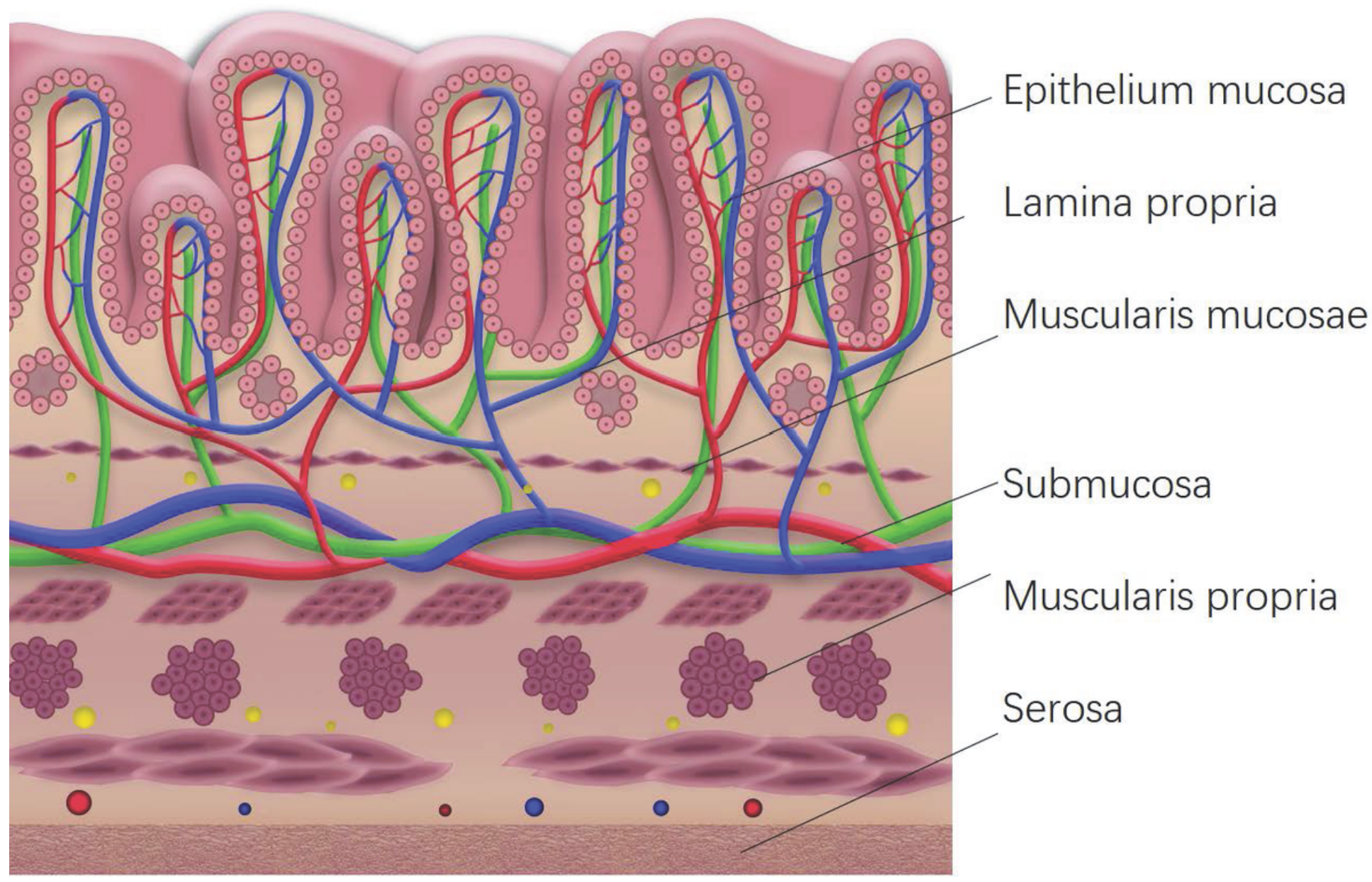

Figure 2

Schematic diagram of the histological structure of the gastric wall, from inside out, consists of the mucosal layer(epthelium+lamina propria +musculairs mucosae), the submucosal layer, the muscularis propria layer and the serosal layer. 


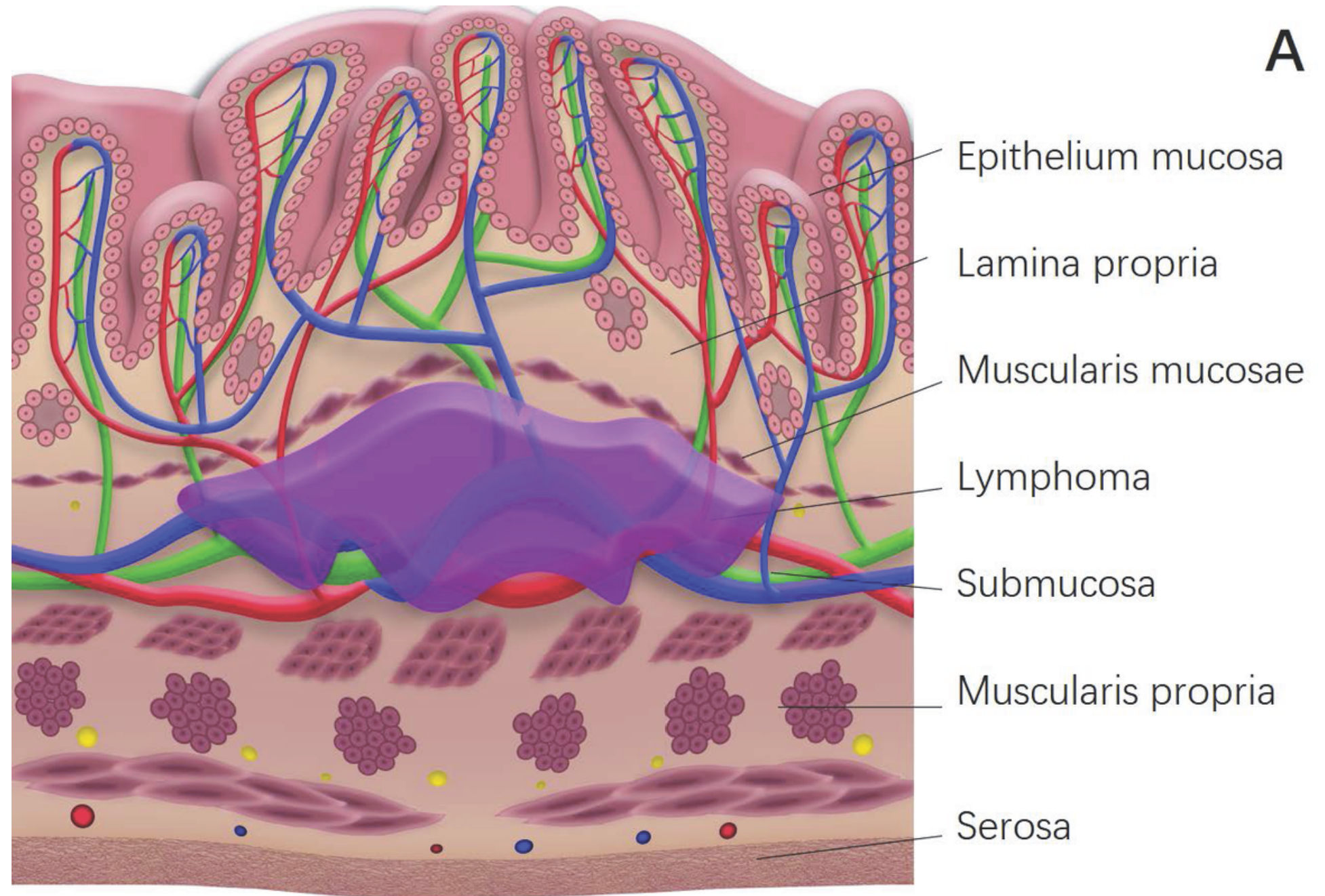

B

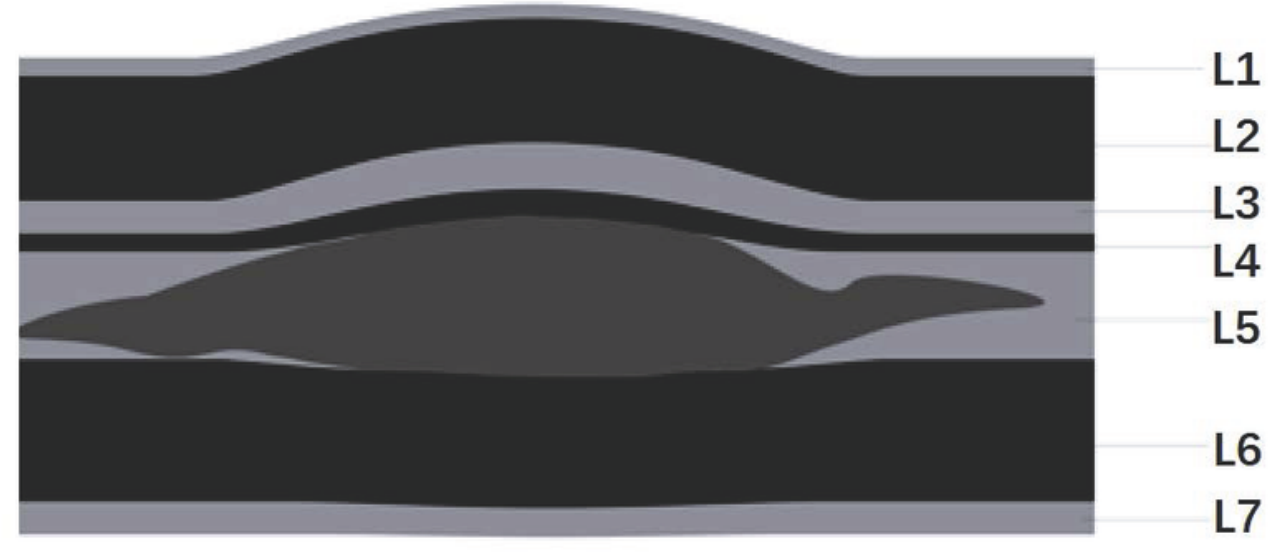

\section{Figure 3}

Diagram of Lymphoma. The gastric wall thickening is consistent with the long axis of the gastric wall. Mainly originate from the submucosa (L5). 


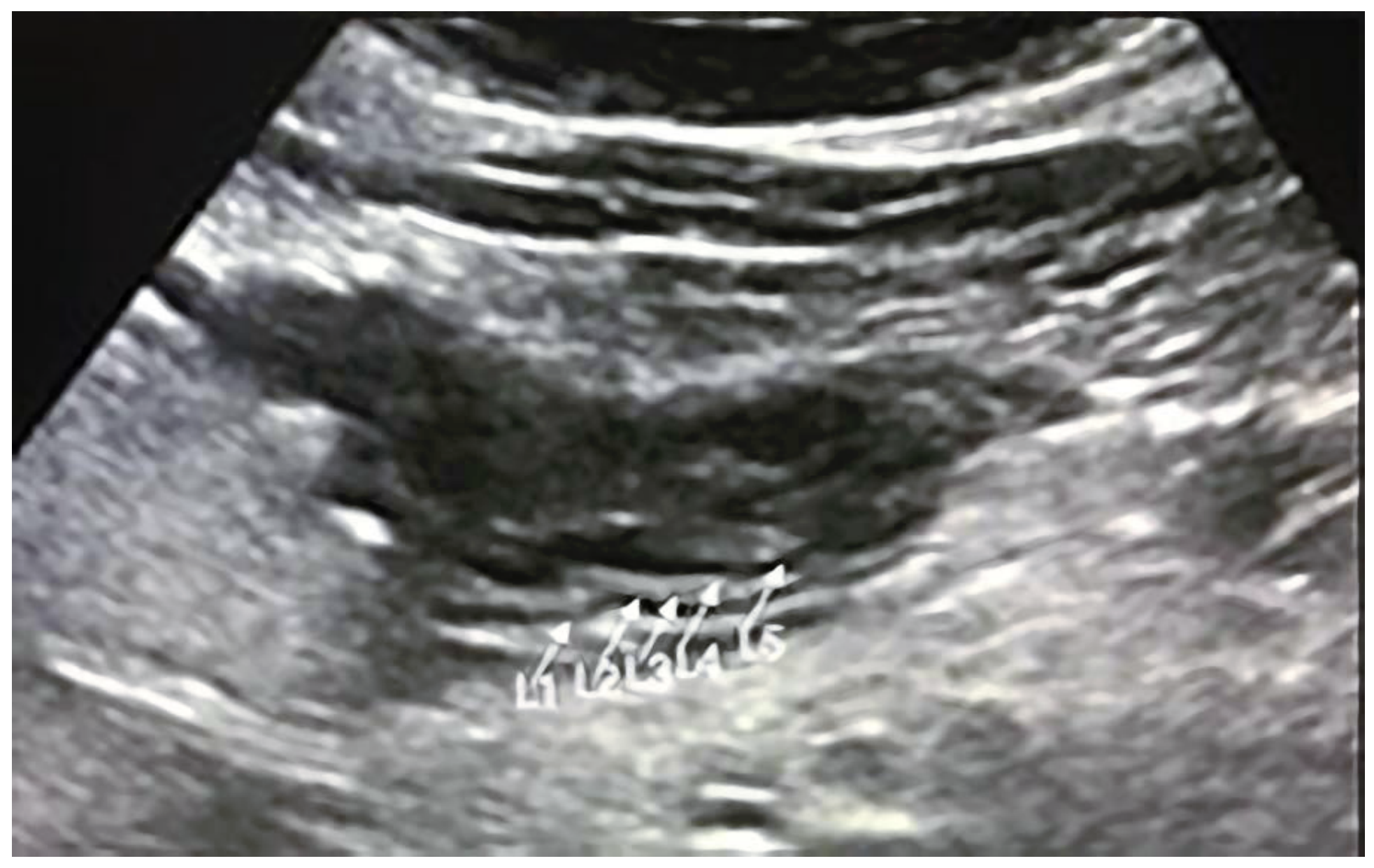

Figure 4

Diffuse large B-cell lymphoma with submucosal (L5) origin. The lesion shows weak-echo in sonograms, and also in line with the long axis of the gastric wall. 


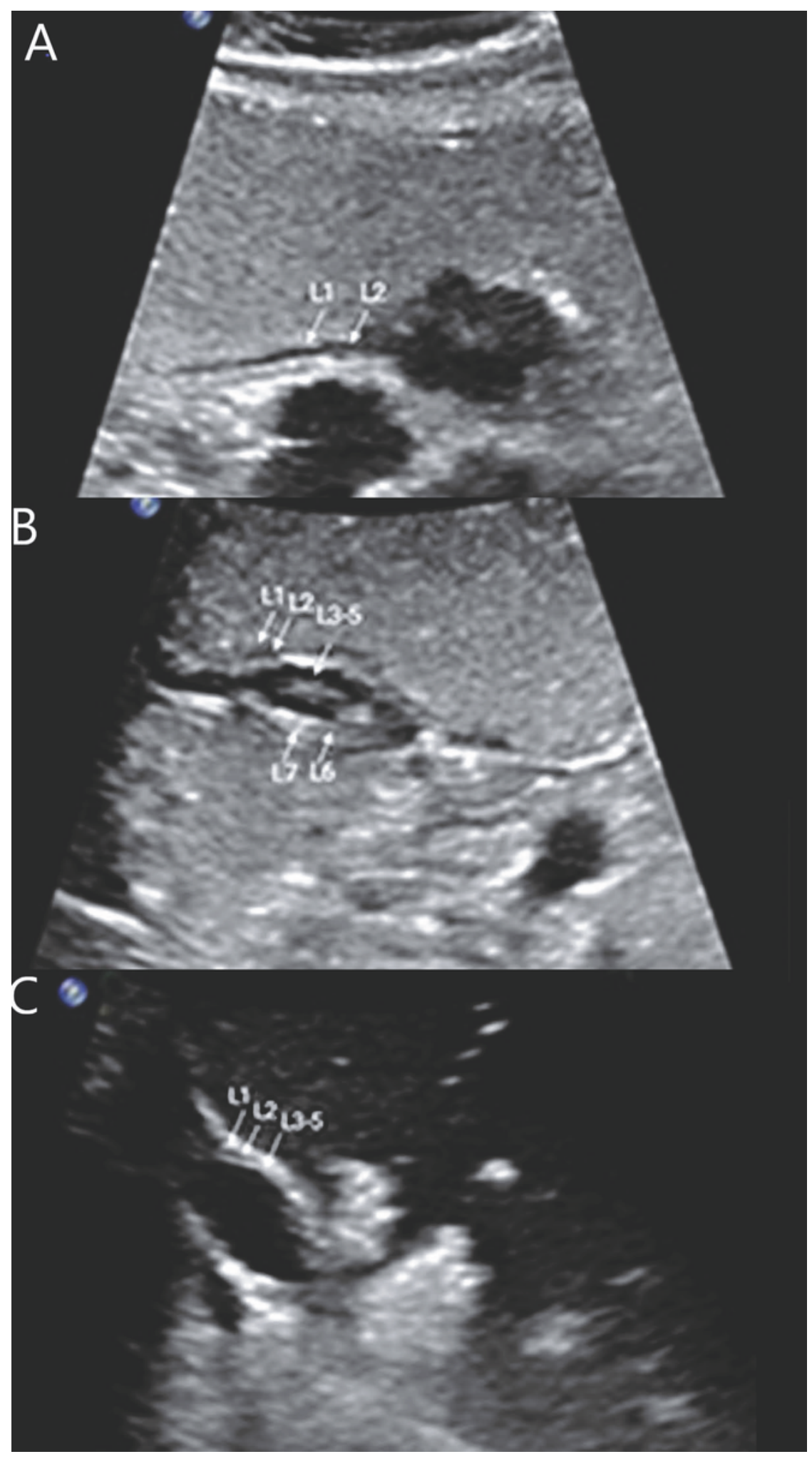

Figure 5

Sonograms of neuroendocrine tumor (A), ectopic pancreas (B), and leiomyoma (C) .(A) Neuroendocrine tumor, closely related to $L 2$, low echo, strong sense of expansion (B) ectopic pancreas, located in $L 5$, flat disk, reticular echo, dot-like strong echo, umbilical-like feature (C)Leiomyoma in L6, in front of mass seen strong-weak-strong echo, internal echo smooth and uniform like silk. 


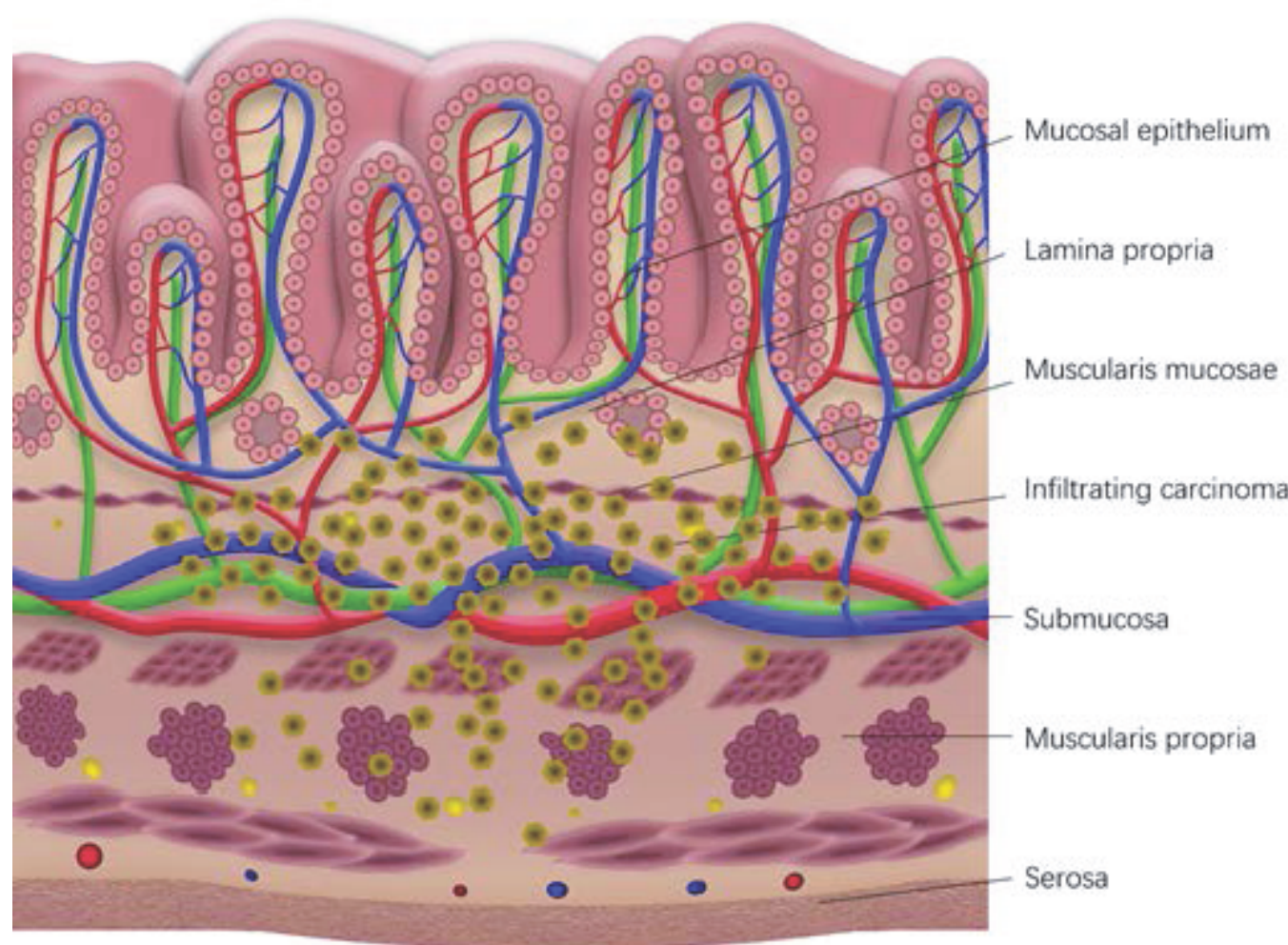

A

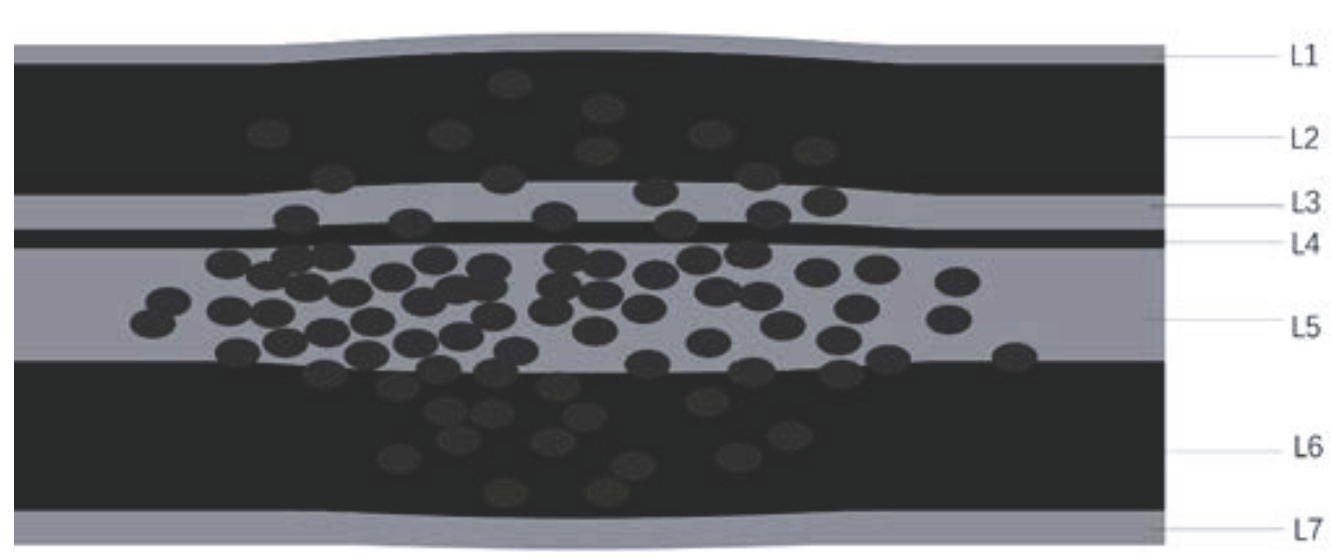

Figure 6

Diagram of diffuse invasive carcinoma. The cancer cells tend to infiltrate under the epithelium of the mucosal surface and grow along the interstitium, no manifestation on the mucosal surface in the early stage. In deeper infiltration cases located in the submucosal layer and muscularis propria (L5, L6). 


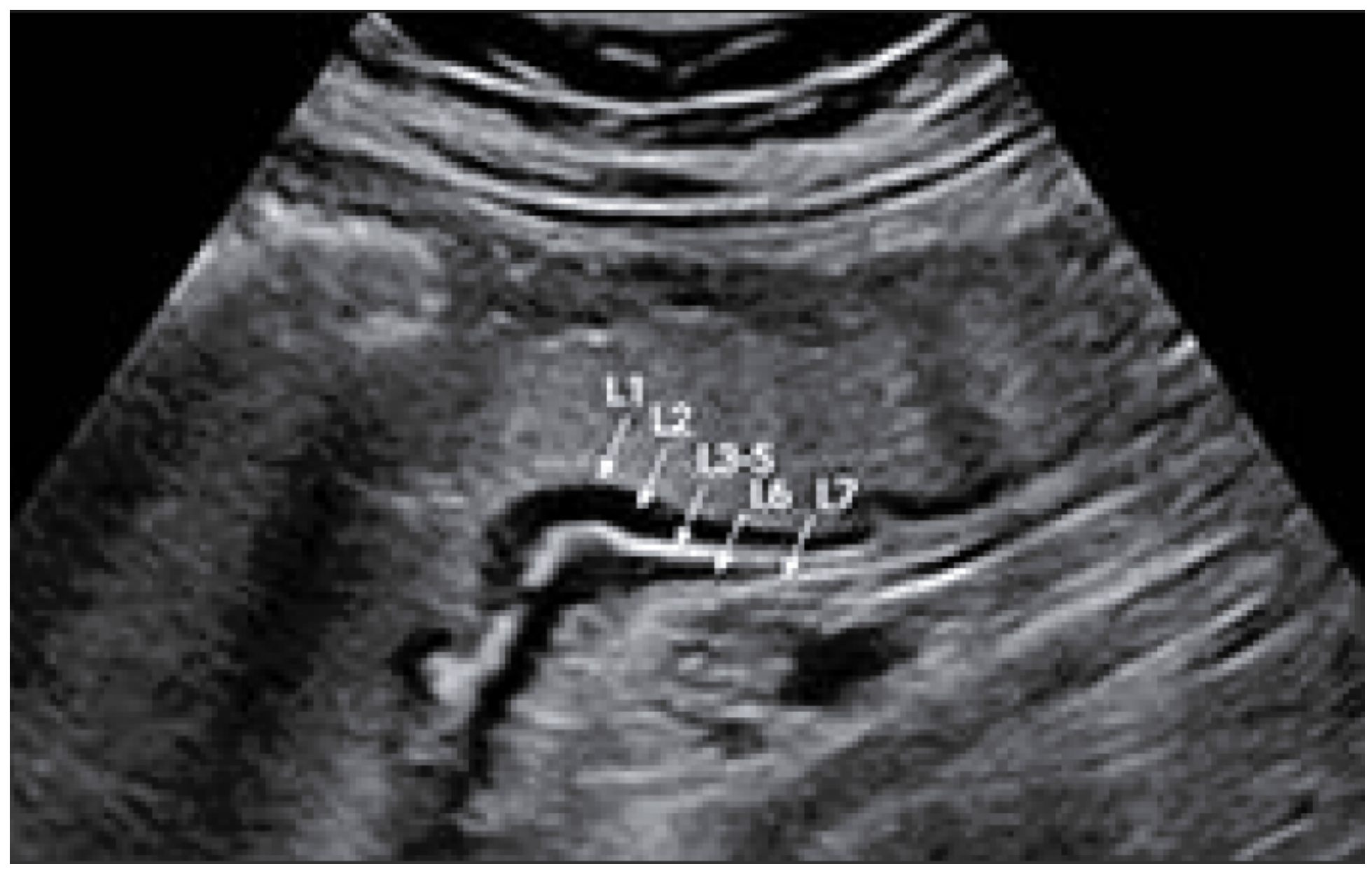

\section{Figure 7}

Diffuse invasive carcinoma in sonograms. L2-L6 involved, seen irregular thickening gastric wall, the thickened is still clear. 